\title{
Studies on conductivity and dielectric properties of polyaniline-zinc sulphide composites
}

\author{
H C PANT, M K PATRA, S C NEGI, A BHATIA, S R VADERA and N KUMAR* \\ Defence Laboratory, Jodhpur 342 011, India
}

MS received 5 February 2005

\begin{abstract}
In the present paper, we report electrical conductivity and dielectric studies on the composites of conducting polyaniline (PANI) with crystalline semiconducting $\mathrm{ZnS}$ powder, wherein PANI has been taken as inclusion and $\mathrm{ZnS}$ crystallites as the host matrix. From the studies, it has been observed that the value of room temperature d.c. conductivity of the composites with volume fraction of PANI $>0.65$ shows an unusual behaviour wherein, conductivity values of the composites exceed that of PANI itself with maximum value as high as 6 times that of PANI at the volume fraction of $0 \cdot 85$. A similar trend has also been observed for the real and imaginary parts of complex dielectric constant values of the composites. This unusual behaviour in the d.c. conductivity and dielectric properties has been attributed to the enhancement in the degree of crystallinity of PANI as a consequence of its interfacial interaction with $\mathrm{ZnS}$ matrix. The results of optical microscopy show coating of PANI all around the $\mathrm{ZnS}$ particles. The temperature dependent conductivity studies suggest the quasi one-dimensional VRH conduction in PANI as well as its composites with ZnS. FTIR and XRD studies have also been reported.
\end{abstract}

Keywords. Complex dielectric constant; percolation threshold; interfacial interaction; degree of crystallinity; characteristic temperature.

\section{Introduction}

Conducting polymers have emerged as a very important class of materials because of their unique electrical, optical and chemical properties leading to the wide range of technological applications. This class of materials provide tremendous scope for tuning of their electrical conductivity from semiconducting to metallic regime by way of doping (MacDiarmid and Epstein 1994; Wessling 1999). The unique properties of conducting polymers not only provide great scope for their applications but also have led to the development of new models to explain their observed properties, particularly various mechanisms of charge transport (Kaiser et al 1995, 1997). Among different conducting polymers, conducting polyanilines are the most extensively studied materials due to the ease of synthesis, better environmental and thermal stabilities and greater scope of playing with chemistry to tailor their properties (Kumar et al 1996). However, when they are taken in the composite form their electrical as well as dielectric properties are altered from those of basic materials. A number of groups have reported studies on the electrical conductivity and dielectric properties of composites of a variety of conducting polymers (Yoon et al 1995; Yang et al 1996; Gangopadhyay et al 2001; Muru-

\footnotetext{
*Author for correspondence (nkjainjd@yahoo.com)
}

gesan and Subramanian 2003). It has been shown that the conductivity of these heterogeneous systems depends on a number of factors such as the concentration of conducting fillers, their shape, size, orientation and interfacial interaction between filler molecules and host matrix (Kryszewaski 1991; Brosseau et al 2001). The geometrical shape of the dispersant governs the ability of conductive network formation which results in large increase in the conductivity (Troung et al 1994). Also, dispersant/matrix interactions and physical properties of the matrix influence the agglomeration of the dispersant phase which, in turn, affects the dielectric properties of the composites. In case of conducting polymers as fillers, the degree of cross-linking between the polymeric chains also affects the electrical properties in these composites (MacDiarmid and Epstein 1995).

Although the percolation theory has been generally used to explain the behaviour of electrical conductivity and dielectric properties of conducting polymer composites (Tuncer et al 2002), but it could not explain very low values of percolation threshold observed in many of the composite systems of conducting polymers. Wessling (1998) proposed a non-equilibrium theory for the composites of conducting polymers. This theory while accounting for the interfacial interactions between conducting polymers and host matrix could explain the observations of low percolation threshold.

In the present paper, we report XRD, IR, optical microscopy, electrical conductivity studies and microwave 
measurements on the polyaniline- $\mathrm{ZnS}$ composite systems to explain the unusual behaviour.

\section{Experimental}

\subsection{Sample preparation}

The samples of conducting polymer-semiconductor composites were prepared by thorough mixing of zinc sulfide powder with conducting $\mathrm{HCl}$-doped emeraldine salt by mechanically grinding them in a pestle mortar while taking weight percentage of conducting polyaniline with an increment of $20 \%$. The composite with $20 \%$ weight fraction of PANI was named as sample, S1 and subsequent composites with concentration of PANI at 40, 60 and $80 \%$ as S2, S3 and S4, respectively. Precise filling factor or volume fraction was calculated by using the following formula

$$
\begin{aligned}
f= & \text { vol. inclusion powder } /(\text { vol. inclusion powder }+ \\
& \text { vol. host powder })(\text { Bober } \text { et al }) \\
= & \left(M_{\mathrm{i}} / \rho_{\mathrm{i}}\right) /\left[\left(M_{\mathrm{i}} / \rho_{\mathrm{i}}\right)+\left(M_{\mathrm{h}} / \rho_{\mathrm{h}}\right)\right],
\end{aligned}
$$

where $M_{\mathrm{i}}, \rho_{\mathrm{i}}\left(=1.52 \mathrm{~g} \mathrm{~cm}^{-3}\right)$ are the mass and density of the inclusion and $M_{\mathrm{h}}, \rho_{\mathrm{h}}\left(=1.96 \mathrm{~g} \mathrm{~cm}^{-3}\right)$ are of host material, respectively.

\subsection{Measurements}

An HP8510C Network Analyser was used to perform transmission and reflection measurements on these samples in X-band $(8 \cdot 2-12.4 \mathrm{GHz})$ of MW frequency. The values of complex permittivity $\left(\varepsilon_{\mathrm{r}}=\varepsilon_{\mathrm{r}}^{\prime}-j \varepsilon_{\mathrm{r}}^{\prime \prime}\right)$ and permeability $\left(\mu_{\mathrm{r}}=\mu_{\mathrm{r}}^{\prime}-j \mu_{\mathrm{r}}^{\prime \prime}\right)$ along with loss tangent $\left(\tan \delta=\varepsilon_{\mathrm{r}}^{\prime \prime} / \varepsilon_{\mathrm{r}}^{\prime}\right)$ of the composite materials were calculated from measured values of $S$-parameters using Nicolson-Ross algorithm (Chen et al 2004). The room temperature d.c. conductivity measurements were performed by using four-point resistivity probe. The variable temperature d.c. conductivity measurements were carried out in the temperature range 20 $300 \mathrm{~K}$ by using four-point resistivity probe placed in Janis Model CCS-850 closed cycle refrigerator system. The XRD spectra of the composites were obtained by using powder XRD system Model PANanalytical X'Pert Pro having $\mathrm{CuK}_{\alpha 1} \mathrm{X}$-ray source of wavelength, $1.5406 \AA$. The FTIR spectra were recorded in $\mathrm{KBr}$ by using SHIMADZU Model 8101A FTIR spectrometer. The optical micrographic studies were carried out by using optical microscope Model Nikon Eclipse E600.

\section{Results and discussion}

\section{$3.1 \quad$ FTIR studies}

The FTIR spectra of the pure materials and their composites are shown in figure 1 . It can be seen from the figure that in case of $\mathrm{ZnS}$ no absorption peaks are observed in the region $4600-400 \mathrm{~cm}^{-1}$. Whereas for pure PANI two prominent absorption peaks are observed at $1713 \mathrm{~cm}^{-1}$ and $1006 \mathrm{~cm}^{-1}$ due to $\mathrm{C}=\mathrm{NH}$ stretching (Lide et al 1993) and terminal monosubstituted benzenes vibration (Harada et al 1989), respectively. Other absorption peaks are observed at $1374 \mathrm{~cm}^{-1}$ due to benzene nitrogen stretching (Lide et al 1993). The absorption peak around $3426 \mathrm{~cm}^{-1}$ are attributed to NH stretching (Harada et al 1989).

Further, it is also observed from the figure that on mixing PANI with $\mathrm{ZnS}$, the IR absorption peak of PANI at $1713 \mathrm{~cm}^{-1}$ due to $\mathrm{C}=\mathrm{NH}$ stretching vibration reduces in intensity as well as broadens showing multiple peaks of weak intensity in the composites, S2, S3 and S4. Furthermore, the intensity of peak around $1006 \mathrm{~cm}^{-1}$ reduces and shifts to higher wave number. The observed changes in the IR spectra of composites are mostly around $\mathrm{C}=\mathrm{NH}$ and terminal monosubstituted benzene sites thereby suggesting strong coupling/interaction of $\mathrm{ZnS}$ crystallites with the imine nitrogen in the conducting polymer.

\subsection{Optical microscopic studies}

The optical microscopic photographs of pure conducting polyaniline, ZnS sample and their composites, S1, S2, S3 and $\mathrm{S} 4$, are shown in figure 2 . From this figure it can be seen that the optical micrograph of pure conducting polyaniline shows independent fine black coloured particles whereas $\mathrm{ZnS}$ particles can be seen as optically transparent particles. Further, it can be seen that in composite S1, where the concentration of PANI is $20 \%$, we observe very thin black layer of PANI around the transparent particles of $\mathrm{ZnS}$. As we increase the concentration of PANI to $40 \%$, the black coating becomes thicker around these particles. On further increasing the concentration of PANI to $80 \%$, we observe that PANI almost completely overcoats the $\mathrm{ZnS}$ particles and they are seen as completely black. The studies, therefore, suggest almost complete and thick coating of PANI around the $\mathrm{ZnS}$ particles at higher concentration of PANI ( $>60 \%)$.

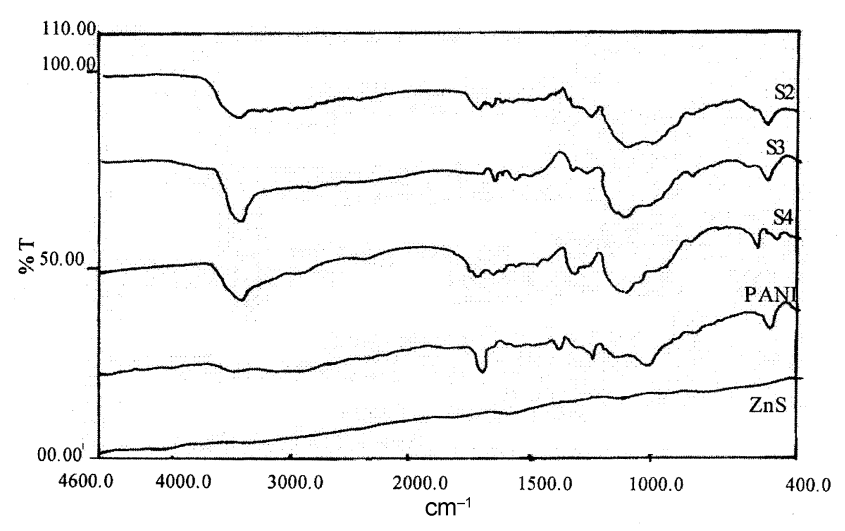

Figure 1. FTIR spectra of PANI, $\mathrm{ZnS}$ and their composites. 

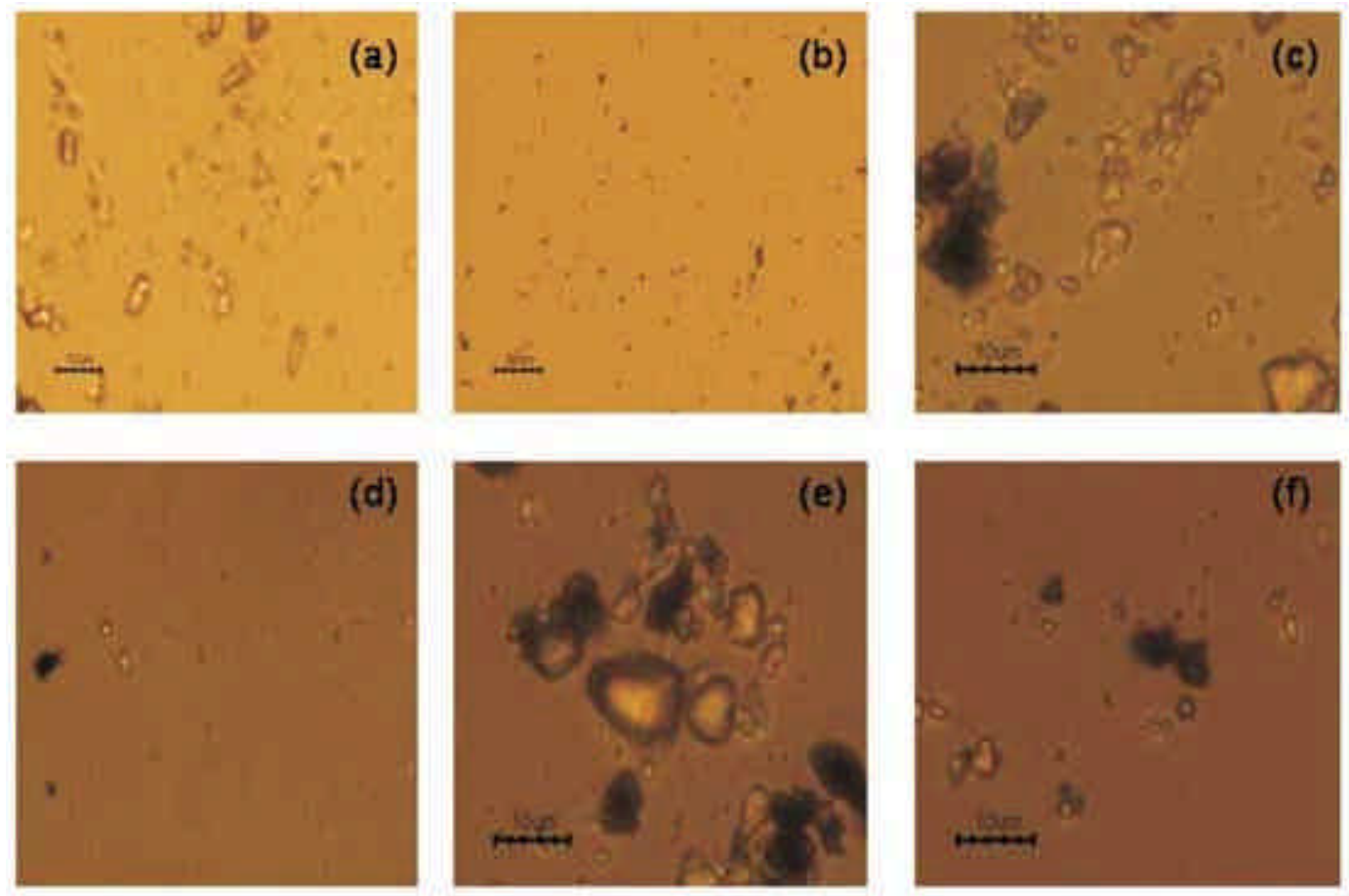

Figure 2. Optical microscopic photographs of (a) ZnS, (b) PANI, (c) S1 (20\% PANI + 80\% ZnS), (d) S2 $(40 \%$ PANI $+60 \% \mathrm{ZnS}),($ e) S3 (60\% PANI $+40 \% \mathrm{ZnS})$ and (f) S4 (80\% PANI + 20\% ZnS).

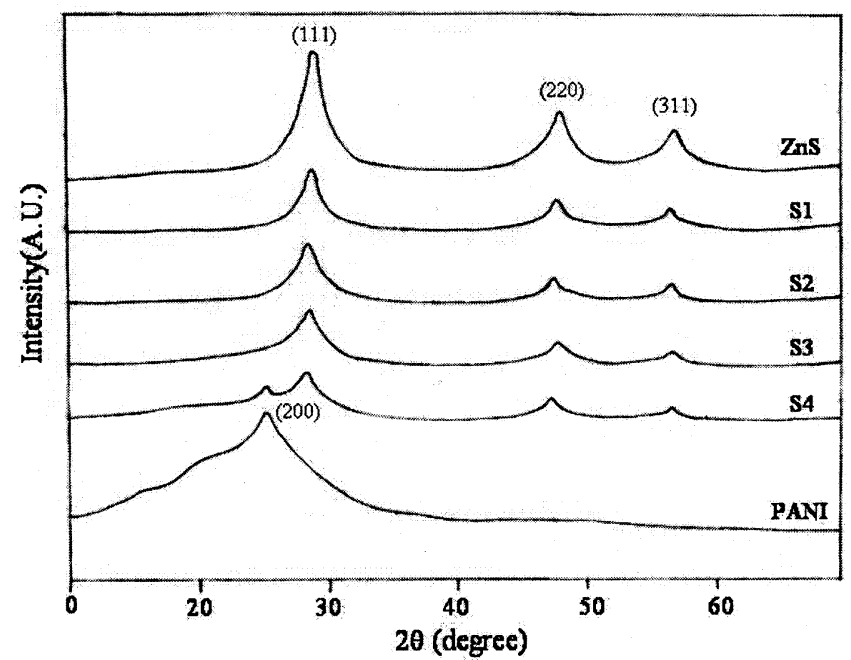

Figure 3. XRD spectra of PANI, $\mathrm{ZnS}$ and their composites.

\subsection{XRD studies}

The powder XRD spectra of pure PANI, pure ZnS and their composites with different volume fractions are shown in figure 3. From this figure, in the XRD spectra of pure PANI, we observe a broad reflection at lower Bragg angle $2 \theta$ value of $25.38^{\circ}$ corresponding to (200) diffraction plane of ES-I structure of $\mathrm{HCl}$ doped PANI (Joo et al 1998). In XRD spectra of $\mathrm{ZnS}$, we observe three reflection peaks at $2 \theta$ value of $28.858^{\circ}, 47.515^{\circ}$ and $56.289^{\circ}$ corres- ponding to (111), (220) and (311) set of diffraction planes, respectively of the cubic crystalline structure.

However, in the XRD spectra of the composites we observe that at $20 \%$ concentration of $\mathrm{ZnS}$ in PANI (sample, S4) intensity of XRD peak due to PANI has been reduced significantly and XRD pattern of $\mathrm{ZnS}$ starts dominating. It is interesting to note that on further increasing the concentration of $\mathrm{ZnS}$ to $40 \%$ (sample, S3) we find peaks due to $\mathrm{ZnS}$ only, even though the composite contains $60 \%$ of PANI. All other samples (samples, S1 and S2) with higher concentration of $\mathrm{ZnS}$ also show XRD patterns due to $\mathrm{ZnS}$ only. XRD study thus suggests that during the mechanical grinding of PANI with ZnS, PANI undergoes interfacial interactions with $\mathrm{ZnS}$ crystallites and looses its own morphology by its coating over $\mathrm{ZnS}$ crystallites.

\subsection{Room temperature d.c. conductivity studies}

The plot of room temperature d.c. conductivity vs volume fraction of PANI-ZnS composites are given in figure 4. It is quite interesting to note that although the conductivity of $\mathrm{ZnS}$ is almost three orders of magnitude lower than that of pure PANI, however, the conductivity values of its composites with PANI above volume fraction, 0.65, are significantly higher than PANI itself (table 1).

In general, percolation theory is used to describe the conductivity behaviour of conducting metal particle filled insulating polymeric composites, wherein, sudden increase in the conductivity of composite is observed above certain 
Table 1. Compositions and d.c. conductivity of PANI-ZnS composites.

\begin{tabular}{|c|c|c|c|c|c|c|}
\hline S1 no. & $\begin{array}{l}\text { Description of } \\
\text { sample }\end{array}$ & $\begin{array}{l}\text { Weight percentage } \\
\text { of constituents }\end{array}$ & $\begin{array}{l}\text { Volume fraction } \\
\text { of PANI }\end{array}$ & $\begin{array}{l}\text { DC conductivity } \\
(\mathrm{S} / \mathrm{cm})\end{array}$ & $\begin{array}{c}\text { VRH } \\
\text { exponent }(\gamma)\end{array}$ & $T_{0}\left({ }^{\circ} \mathrm{K}\right)$ \\
\hline 1. & Pure ZnS & $100 \% \mathrm{ZnS}$ & $0 \cdot 00$ & $0 \cdot 0002$ & - & - \\
\hline 2. & Composite, S1 & $20 \%$ PANI $+80 \% \mathrm{ZnS}$ & $0 \cdot 25$ & 0.0022 & $0 \cdot 40$ & $4929 \cdot 5$ \\
\hline 3. & Composite, S2 & $40 \%$ PANI $+60 \% \mathrm{ZnS}$ & $0 \cdot 45$ & 0.0053 & 0.41 & $4512 \cdot 5$ \\
\hline 4. & Composite, S3 & $60 \%$ PANI $+40 \% \mathrm{ZnS}$ & $0 \cdot 65$ & 0.4954 & 0.47 & $2562 \cdot 0$ \\
\hline 5. & Composite, S4 & $80 \%$ PANI $+20 \% \mathrm{ZnS}$ & $0 \cdot 85$ & $0 \cdot 8088$ & $0 \cdot 50$ & $1600 \cdot 6$ \\
\hline 6. & Pure PANI & $100 \%$ PANI & $1 \cdot 00$ & $0 \cdot 1367$ & 0.52 & $2712 \cdot 0$ \\
\hline
\end{tabular}

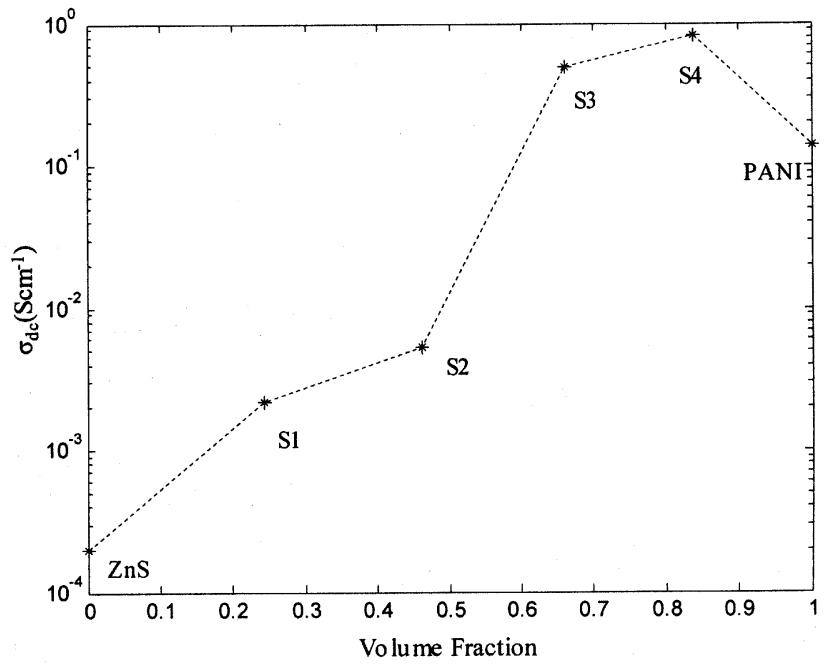

Figure 4. Plot of d.c. conductivity vs volume fraction in PANI-ZnS composites.

concentration of conducting fillers which is called 'percolation threshold'. On further increasing the concentration of conducting filler above percolation threshold, the conductivity values gradually increase and attain the value of filler at volume fraction of unity. The classical percolation theory has successfully explained the conductivity behaviour of conducting polymer filled insulating polymeric composites having higher values of percolation threshold, $>0 \cdot 16$. However, it alone could not explain the conductivity behaviour at very low values of percolation threshold observed in a number of conducting polymers based systems (Fizazi et al 1990; Suzuki et al 1990; Blaszkiewicz et al 1992). This is due to the fact that the percolation theory is purely statistical in nature and does not account for the interfacial interactions of conducting polymers with their matrices. Wessling (1998) carried out extensive studies on the conductivity behaviour of dispersions of different types of conducting polymers in a variety of polymeric matrices and attributed the lower values of percolation threshold of these composites to the interfacial interactions between the filler and host matrix. From their studies, they proposed a non-equilibrium theory based on the thermodynamics of interfacial interactions to explain the conductivity behaviour of heterogeneous conducting polymer composites. In all such studies the conductivity is maximum for conducting filler. However, in the present studies, the conductivity values of the composites have been found to be greater than pure PANI and the same cannot be explained on the basis of non-equilibrium theory.

The mechanisms of electrical conductivity in conducting polymers are well studied. It has been reported in the literature that the d.c. conductivity of conducting polymers depend on their morphology and certain other factors such as type of monomer, doping level, degree of crystallinity etc (MacDiarmid and Epstein 1994). It has been reported that the large increase in conductivity originates from the fact that doped (protonated) polyaniline is a polyelectrolyte, i.e. a macromolecule bearing a large number of ionizable groups. MacDiarmid and Epstein (1995) reported that its molecular conformation upon reaction with the vapour of appropriate substance changes the compact coil structure to an expanded coil like structure. The attainment of expanded molecular conformation acts to reduce $\pi$-conjugation defects in the polymer backbone and the opening up of coil tends to promote linear conformation necessary for crystallization, thus increasing the crystallinity of the polymer with enhancement of the intermolecular component of the bulk conductivity.

In the present composite systems we feel that the mechanical grinding of PANI with $\mathrm{ZnS}$ causes strong interfacial interactions between PANI and ZnS crystallites, as suggested by the FTIR and XRD studies, thereby changing the molecular conformation of PANI from compact coil structure to an expanded coil like structure. As a consequence there may be an enhancement in the conductivity of composites of PANI and $\mathrm{ZnS}$. Further, at higher volume fractions of PANI, it fully overcoats $\mathrm{ZnS}$ particles and the conductivity of composites attains values greater than pure PANI, as has been observed in the optical microscopic photographs of composites, S3 and S4.

\subsection{Temperature dependent d.c. conductivity studies}

The plot of d.c. conductivity, $\sigma_{\text {d.c. }}$ of conducting PANI and its composites with temperature is shown in figure 5. From this figure we observe the exponential increase in $\sigma_{\text {d.c. }}$ with temperature from $20-300 \mathrm{~K}$ in pure PANI and its composites, thereby indicating the behaviour of disordered 
semiconductor. Further, composites, S3 and S4, maintain their higher $\sigma_{\text {d.c. }}$ values than pure PANI over the entire temperature range $20-300 \mathrm{~K}$.

The analysis of temperature dependent conductivity data suggests that the charge transport mechanism in PANI as well as all its composites can be explained by the variable range hopping (VRH) model where dependence of d.c. conductivity is governed by the following relation (Dutta et al 2001),

$$
\sigma_{\text {d.c. }}(T)=\sigma_{0} \exp \left(-\frac{T_{0}}{T}\right)^{\gamma},
$$

where $T_{0}$ is the characteristic temperature evaluated from linear slope of $\ln \left(\sigma_{\text {d.c. }}\right)$ vs $T^{-\gamma}$ as shown in figure 6 (table $1), \sigma_{0}$ the d.c. conductivity at absolute zero temperature, and $\gamma$ the VRH exponent which depends upon the dimension

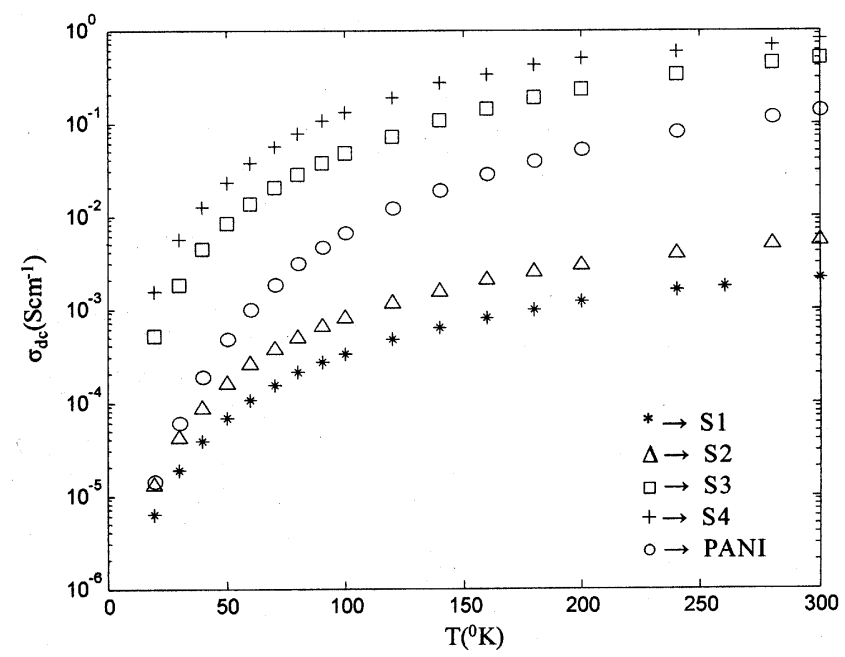

Figure 5. Plot of d.c. conductivity vs temperature of PANI$\mathrm{ZnS}$ composites.

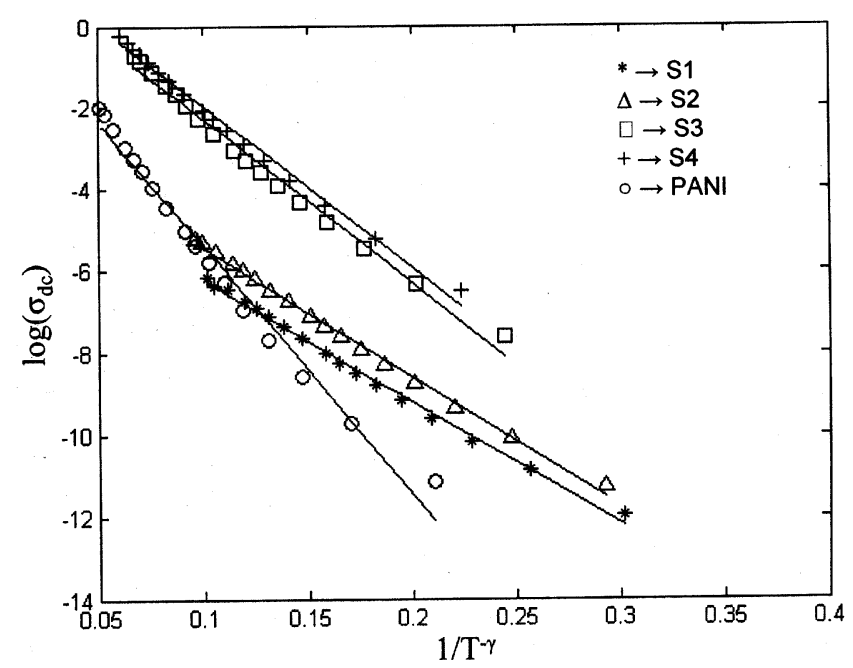

Figure 6. Plot of $\log \left(\sigma_{\text {d.c. }}\right)$ vs $T^{-\gamma}$ of PANI-ZnS composites. of the system. The value of VRH exponent, $\gamma$, has been determined from the reduced activation energy $(W)$,

$$
W=\frac{d \ln \sigma}{d \ln T},
$$

and it has been found to lie between 0.40 and 0.51 (table 1), which indicates the presence of quasi one-dimensional hopping structure in all the composites similar to that of pure PANI (Epstein et al 1994). Hence, these studies show that although the composites, S3 and S4, having higher values of $\sigma_{\text {d.c. }}$ as compared to pure PANI but formation of 3-dimensional conducting network is not likely and thus such enhancement of $\sigma_{\text {d.c. }}$ values can be attributed to the uncoiling of polymeric chains due to strong interfacial interactions between $\mathrm{ZnS}$ crystallites and PANI caused by mechanical grinding.

\subsection{Microwave characterization}

The plots of complex permittivity and loss tangent vs volume fraction of PANI-ZnS composites at $10 \cdot 3 \mathrm{GHz}$ are given in figure 7 . From the figure it is observed that these composites show high values of both real $\left(\varepsilon_{\mathrm{r}}^{\prime}\right)$ and imaginary $\left(\varepsilon_{\mathrm{r}}^{\prime \prime}\right)$ parts of dielectric constant. Moreover, the values of loss tangent $(\tan \delta)$ are quite high, suggesting that they are highly lossy materials. Further, it is observed that the values of dielectric constant follow the trend almost similar to the one in the case of d.c. conductivity wherein the values of dielectric constant of composites, S3 and $\mathrm{S} 4$, are higher as compared to pure PANI, which can be attributed to the higher values of electrical conductivity of these composites.

In general, in case of heterogeneous mixtures, the behaviour of dielectric properties is governed by Maxwell-
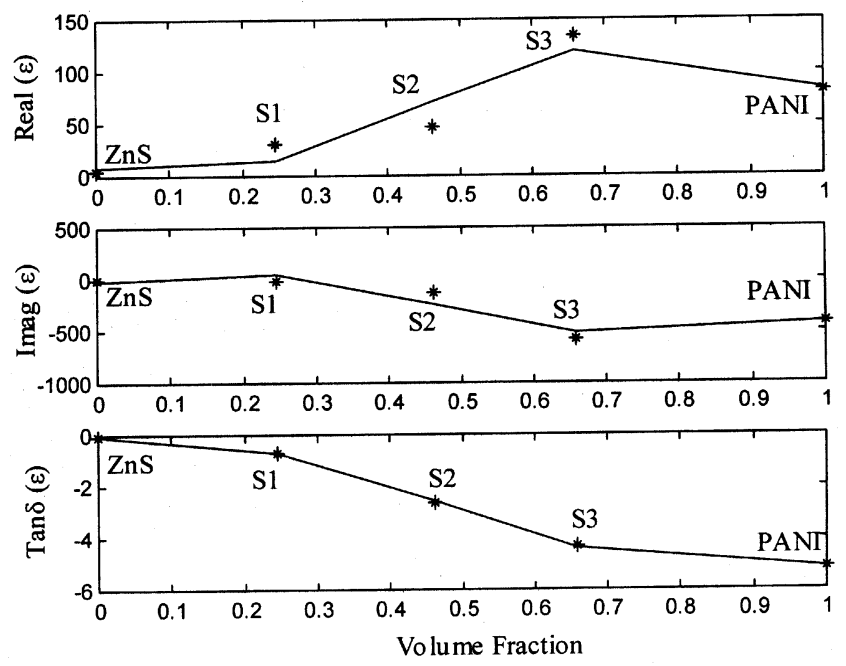

Figure 7. Plots of complex permittivity and $\tan \delta$ vs volume fraction of PANI-ZnS composites at $10 \cdot 3 \mathrm{GHz}$. 
Wagner-Siller model (Hedying 1977). As per this model, it is predicted that the values of dielectric constant increase with the increase in the concentration as well as conductivity of the inclusion. Although, this model takes into consideration the interfacial polarization between the inclusion and the host particles, but the results of present study could not be explained by it. Also, effective medium theory (EMT) (Merrill et al 1999) has also been mostly used to explain the effective dielectric properties of composites, but the results of our studies could not be explained by the same, as our system has been rendered complex due to interactions between PANI and $\mathrm{ZnS}$ resulting into modification in morphology of PANI.

\section{Conclusions}

From the above studies, we have observed that the values of d.c. conductivity and dielectric constant of PANI have unusually enhanced by simply mechanically grinding it with crystalline ZnS powder. The FTIR studies suggest the occurrence of chemical interaction between PANI and $\mathrm{ZnS}$ crystallites, while the optical microscopic studies indicate complete overcoating of PANI on $\mathrm{ZnS}$ crystallites at volume fraction $>0 \cdot 65$. The XRD study suggests modification in the structure of PANI due to its interfacial interactions with $\mathrm{ZnS}$ crystallites. The enhancement in the conductivity has been attributed to the increase in the degree of crystallinity of PANI due to uncoiling/stretching of polymeric chain caused due to the strong interfacial interactions with $\mathrm{ZnS}$ crystallites. The temperature dependent conductivity studies suggest the quasi onedimensional VRH conduction in pure PANI and its composites. These composites have been found to show high values of both real and imaginary parts of dielectric constant and loss tangent, thus having good microwave lossy behaviour.

\section{References}

Blaszkiewicz M, McLachlan D S and Newnham R $1992 J$. Polym. Eng. Sci. 32421

Bober K N, Giles R H and Waldman J, Tailoring the microwave permittivity and permeability of composite materials; Internet site: http://stl.uml.edu/PubLib/Bober,\%20Tailor\%20Micro\% 20Perm.pdf

Brosseau C, Queffelec P and Talbot P 2001 J. Appl. Phys. 89 4532

Chen L F, Ong C K, Neo C P, Varadan V V and Varadan V K 2004 Microwave electronics: Measurement and material characterization (England: John Wiley \& Sons) p. 177

Dutta P, Biswas S, Ghosh M, De S K and Chatterjee S 2001 Synth. Met. 122455

Epstein A J et al 1994 Synth. Met. 65149

Fizazi A, Moulton J, Pakbaz K, Rughooputh S D D, Smith P and Heeger A J 1990 Phys. Rev. Lett. 642180

Gangopadhyay R, De A and Ghosh G 2001 Synth. Met. 123529

Harada I, Furukawa Y and Ueda F 1989 Synth. Met. 29303

Hedying P 1977 Dielectric spectroscopy of polymers (Bristol: Helger) p. 28

Joo J, Long S M, Pouget J P, Oh E J, MacDiarmid A G and Epstein A J 1998 Phys. Rev. B57 9567

Kaiser A B, Subramanium C K, Gilberd P W and Wessling B 1995 Synth. Met. 69197

Kaiser A B, Liu C J, Gilberd P W, Kemp N T, Wessling B, Partridge A C, Smith W T and Shapiro J C 1997 Synth. Met. 84699

Kryszewaski M 1991 Synth. Met. 45289

Kumar N, Vadera S R, Singh J, Das G, Negi S C, Aparna P and Tuli A 1996 Def. Sci. J. 5691

Lide D R and Frederikse H P R 1993 Handbook of chemistry and physics (Boca Raton, Florida: CRC Press) pp 9-153

MacDiarmid A G and Epstein A J 1994 Frontiers of polymers and advanced materials (New York: Plenum Press) p. 251

MacDiarmid A G and Epstein A J 1995 Synth. Met. 6985

Merrill W M, Diaz R E, LeRe M M, Squires M C and Alexopoulos N G 1999 IEEE Trans. Antenna Propg. 47142

Murugesan R and Subramanian E 2003 Bull. Mater. Sci. 26 529

Suzuki Y, Heeger A J and Pincus P 1990 Macromolecules 23 2180

Troung V T, Codd A R and Forsyth M 1994 J. Mater. Sci. 29 4331

Tuncer E, Serdyuk Y V and Gubanski S M 2002 IEEE Trans. Dielec. Electr. Insul. 9809

Wessling B 1998 Handbook of conducting polymers (New York: Marcel Dekker Inc. Press) p. 471

Wessling B 1999 Synth. Met. 1021396

Yang J, Hou J, Zhu W, Xu M and Wan M 1996 Synth. Met. 80 283

Yoon C O, Reghu M, Moses D, Cao Y and Heeger A J 1995 Synth. Met. 26255 